This paper reports a theoretical study into the saze-typegrate section of a large litter cleaner in mounted cleaners that operate on a cotton harvester, as well as the theoretical and experimental justifications for its parameters. The effect exerted by a mounted cleaner on the process of cleaning raw cotton when processing in a cotton gin has been studied.

A theoretical model of the impact of grates on weeds in cleaning processes has been developed. A condition for repelling the litter by grates and removing it from a cotton technological flow has been studied. The use of grates with a flat-shaped front edge allows for a steady reduction in the amount of damage in the raw cotton fiber, which improves the fiber quality and leads to a decrease in the number of defects and debris.

The experimental and theoretical studies have produced evidence that enables the efficient operation of mounted-type cleaning machines in the cotton-cleaning industry.

The movements of raw cotton as a viscoelastic body at the free impact of litter with the teeth of the saw against a stationary surface of the grate were investigated; the force schemes between the grates and saws were considered. The effect of a saz-type drum on the technological properties of raw cotton was investigated, namely on seed damage and the formation of the free fiber.

$A$ model of interaction between weed particles and grates was considered; the trajectories of the litter flight were shozon in the function of the slope of the grate and the recovery factor.

The issues of the relationship between the physical-mechanical properties of raw cotton, the elastic characteristics of raze cotton, and the impact force of cotton flies against the grate with a flat working face. Solving these issues could make it possible to determine the optimal structure of the raw cotton cleaning mechanisms, which would improve the effectiveness of cleaning raw cotton from weeds.

Based on the identified functional links, it has become possible to construct new or improve existing structures of the sawtype grate section of mounted cleaners. Practical experience shows that the use of the designed structure in large litter cleaners of the mounted type produces a significant increase in the cleaning effect of the machine

Keywords: mounted cleaner, large litter, grate, seed damage, free fiber, cleaning effect
DOI: $10.15587 / 1729-4061.2021 .229032$

\section{THEORETICAL STUDY OF THE GRATE-SAW- TYPE LARGE-LITTER CLEANER OF THE MOUNTED TYPE}

\author{
Husnu Kadir Karimov \\ Senior Lecturer
}

Department of Light Industry Engineering and Design

University of Technology of Azerbaijan (UTECA)

Sh. I. Khatai ave., 103, Ganja, Azerbaijan, AZ 2011

E-mail: kerimov_husnu@mail.ru

Es mira Mustafayeva

Doctor of Philosophy in Mathematics*

E-mail:m_esmira@unec.edu.az

EIman Jafarov

$\mathrm{PhD}$, Associate Professor*

E-mail: elman.cafarov.73@mail.ru

Terane Safarova

$\mathrm{PhD}$, Senior Lecturer*

E-mail: tarana.safarova1@mail.ru

Fazil Veliev

Doctor of Technical Sciences, Professor*

E-mail: fazil-uzbekr@mail.ru

*Department of Engineering and Applied Sciences Azerbaijan State Economic University (UNEC) Istiglyaliyat str., 6, Baku, Azerbaijan, AZ 1001
Received date 10.03.2021

Accepted date 12.04.2021

Published date 26.04.2021
How to Cite: Karimov, H., Mustafayeva, E., Jafarov, E., Safarova, T., Veliev, F. (2021). Theoretical study of the grate-saw-type large-litter cleaner of the mounted type. Eastern-European Journal of Enterprise Technologies, 2 (7 (110)), 74-84. doi:https://doi.org/10.15587/1729-4061.2021.229032
1. Introduction

The interaction between the working bodies of a sawtype grate section and the processed raw cotton product largely determines the efficiency of cleaning machine operation. However, when the fiber material hits the grate, in some cases, along with the intensive removal of litter and crushing of technical complexes (when processing thin cotton), mechanical damage to cotton elements may occur. This negatively affects their spinning properties. Therefore, investigating the process of impact interaction is of interest both from the point of view of the mechanics of weed particle removal and from the manufacturing point of view - obtaining better raw materials with less loss of spinning fibers.

The pre-cleaning section of raw cotton from large weeds is essentially an element of a large litter cleaner of the mounted type built into the cotton harvester. Pre-cleaning raw cotton in mounted cleaners could improve the operation of cleaning machines, which are part of the regulated technological process.

Devising a theory of raw cotton behavior at impact and using the basic patterns of mechanics in relation to the manufacturing processes of primary cotton processing in large litter cleaners of the mounted type has made it possible to experimentally determine the force of a cotton fly's impact against the grate with a flat working face. Thus, all cotton harvesting machines for medium-fiber raw cotton are not equipped with a satisfactory structure of the cotton cleaner. Reducing the amount of weeds in harvested raw cotton, which would shorten the technological process of its cleaning at cotton mills, is a relevant task whose solution is predetermined by the accelerating scientific and technological progress in the production and processing of raw cotton. 


\section{Literature review and problem statement}

The first theoretical and experimental justifications for the parameters of the large litter cleaner were given in works [1,2]. Their authors revealed the physical essence of throwing raw cotton onto the drum's saw-type surface and fixing it with brushing devices. However, there is no broad description of all the factors that influence the cleaning process; the general methodology for studying saw-type cleaners is set out.

Paper [3] reports the result of studying the cleaning process, treating raw cotton fibers and seeds on cleaning machines while subjected to multiple dynamic influences. However, the study was not carried out properly in terms of selecting the frequency of the system's natural vibrations, and, at a minimum, the impact force values derived in the cited paper are an order of magnitude higher than those that are actually observed.

The most profound and significant theoretical and experimental studies to substantiate the parameters of capturing elements, grate bodies, the diameter and speed of drums are described in work [4]. Experiments have shown that with the increase in the speed of the drum at an equal number of grates the cleaning effect increases but the crushing of seeds and the amount of free fiber increases dramatically.

Paper [5] provides the basics of the mechanics of the process of interaction between cotton-raw flies and the working bodies in the section of large litter cleaner. The authors investigated the processes of fixing and removing raw cotton from the saw-type gear, as well as the pulses of impact interaction between the flies and the grate. At the same time, the transition to the laws of deformation and change of shape, the volume of raw cotton under the influence of static and dynamic loads have not been investigated.

Paper [6] mainly aimed at optimizing individual nodes in a grate-saw-type section but addressed the theoretical and experimental justification of the profile, the inclination angle of the front face, and other indicators of the drum's teeth, as well as comparative tests of different teeth. The application of cleaners made with such a drum increased the removal of fibrous material with weeds.

Study [7] considered the choice of an optimal profile of the saw's tooth in terms of improving the cleaning effect while reducing the amount of free fiber and seed damage. A formula has been proposed to determine the distance between the rows of saws and the pitch of teeth in large litter cleaners.

Based on studies reported in $[6,7]$, a distance of $12.5-13 \mathrm{~mm}$ between the rows of teeth was recommended. It has been established that a decrease in the tooth profile leads to an increase in the fragmentation of seeds while the increase negatively affects the technological reliability (capture of flies) and the cleaning effect of the machine.

It was found in [8] that the construction of cleaners for medium-fiber raw cotton did not take into consideration the possibility of cleaning thin-fiber raw cotton, which has a lower strength of attaching fiber to seeds. In order to clean the thin-fiber raw cotton, such speed modes must be found for the cleaner at which the natural physical and mechanical properties of fiber and seeds would be preserved.

A series of studies $[9,10]$ investigate the fixing of cotton flies on the drum gear; there are recommendations for installing two fixed brushes to ensure that the fiber material is placed satisfactorily on the gear. The theoretical justification of the brush was given, taking into consideration the emerging forces based on the theory of changes in the amount of movement, taking into account elastic properties. Based on the practical application of American cleaners, elongated brushes were recommended. However, as shown by comparative tests, these brushes demonstrate worse fixing properties, which negatively affects the technological reliability (capture of flies) and the cleaning effect of the machine.

Much attention in the cited works, especially [11, 12], is paid to the impact process of the interaction between the fly and the grate, as well as the process of separating the litter from the fly. Based on the theorem of change in the amount of motion, a formula was derived to determine the difference in the impact pulse, explaining the process of separating litter from the fibrous mass. However, the main drawback of the cited studies is obvious - the essence of the impact was not defined, therefore, it is impossible to estimate such important parameters as the force and time of impact.

The comprehensive study covers the full range of problems, and it is impossible to dwell on all in detail. The theory of fly's impact against working bodies, the synthesis and analysis of grates, experimental determination and experimental-theoretical assessment of impact parameters have not been studied. The issues of contact fiber destruction, the matrix prediction of process technology, new ways, and cleaning methods are still to be examined.

Thus, up to now, the issue related to studying a gratesaw-type large litter cleaner of the mounted type has remained unexplored.

\section{The aim and objectives of the study}

The aim of this work is to study the effect of a grate-sawtype large litter cleaner of the mounted type on the technological process of cleaning raw cotton during processing in a cotton gin. This would reduce the amount of litter in the harvested raw cotton that could shorten the process of its cleaning in cotton gins.

To accomplish the aim, the following tasks have been set:

- to build a theoretical model of the influence exerted by grates on weeds in cleaning processes;

- to assess the dynamic characteristics of the saw-type grate section;

- to determine the influence of the inclination angle of the grate on its cleaning effect.

\section{Procedure to study the process of cleaning raw cotton from weeds using mounted cleaners}

The theoretical part of this study on selecting the inclination angle of grates and the condition for repelling weed particles in order to effectively remove them from a technological flow is based on the application of a generalized model of the cleaning process.

A gentle technology has been chosen that makes it possible to fully enough preserve the natural properties of cotton while reducing the natural fading of process efficiency.

The selection of an actual drum cross-section has been scientifically justified; the dependence of the cleaning effect exerted by working bodies on the cleaned raw material has been refined. 
We have experimentally determined the impact force of cotton flies against a flat grate and obtained the general differentiated characteristics of the cleaning process in the grate-saw-type section under the raw cotton harvesting conditions and at a laboratory bench.

The experimental results have been treated by the methods of mathematical statistics and compared with theoretical models. It is shown that the inevitable fading of cleaning process efficiency in the interaction with subsequent similar working bodies can be eliminated by changing the direction and force of the impact.

The rational choice of an actual cross-section of the drum has allowed it to be effectively used as a separating working body while creating a gentle environment for cotton to interact with the saw-type gear. A new procedure for calculating the cleaning effect for each of the cleaner's litter-repelling elements has been devised, allowing for an objective assessment of the participation of each element in the cleaning process. The cleaner's grate is designed on the basis of the study of the impact process and the conditions for the most favorable removal of litter from the technological flow.

In order to simulate the process of transporting raw cotton directly in a cotton harvester, a special laboratory set-up (Fig. 1) was designed, equipped with a centrifugal fan with an elliptical inlet. The set-up provides for the possibility of changing the performance of the installation, gaps between the grates, and their inclination angles. The designed set-up makes it possible to simulate the cleaning process according to the chosen scheme in a cotton harvester.

Fig. 1 shows the diagram of the laboratory set-up.

The installation includes storage bunker 1 to be filled with a certain amount of raw cotton. The raw material in the bunker falls into the gap between feed rollers 2 , which, rotating towards each other, capture the raw cotton and feed it into pipeline 3 , which has a valve to regulate the amount of air absorbed by fan 4 , as well as the speed of the airflow to the cleaner.

One of the axes of the feed roller is equipped with a variator to regulate its number of revs, thus the performance of the cleaner. Next, the raw cotton is sucked by the air stream created by fan 4, pumped into pipeline 5 of the rectangular cross-section, then, by hitting it and gliding over brush 7 , falls on saw-type-perforated drum 6 .

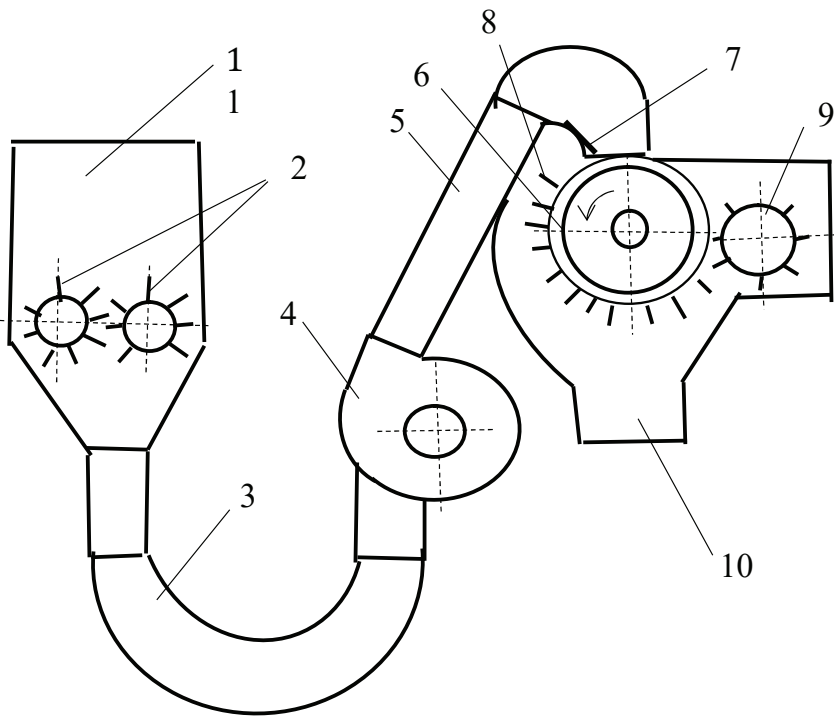

Fig. 1. Diagram of the laboratory set-up
The saw-type-perforated drum is assembled of a segmented reinforced saw. The drum revolves around a pipe that, being an axis, simultaneously serves as a partition inside the drum. At the same time, the air with small weed impurities passes through the gap between the saw-type gear and is discharged outwards, while the raw cotton, picked up by the teeth of saw-type-perforated drum 6, is leveled and wound with still brush 7 . Then the slices and flies from the raw cotton, grabbed by the teeth of the saw-type-perforated drum, are subjected, when rotating, to repeated impact-shaking influence from grates 8 , installed in a special sector with the help of brackets that make it possible to adjust the gap and inclination angle of the grates.

As a result of shaking, the bond between the raw cotton and litter is weakened, the weeds fall through the gaps between the grates into garbage can 10. The raw cotton from the teeth of saw-type-perforated drum 6 is removed by removable drum 9.

The designed set-up makes it possible to simulate the cleaning process according to the chosen scheme in a cotton harvester.

Current studies into impact processes mainly consider the impact of a single fly against a grate. The force of impact is measured using a tubular sensor, the procedure for which is reported in [3]. The experiments that involved the cotton-raw variety "T-1", machine-harvested, grade 1 , with a moisture content of $7.8 \%$, were performed according to the following procedure: a cotton fly was fixed on the saw-type drum, with a length of the link between the fly and the drum of $20 \mp 0.5 \mathrm{~mm}$. Rotating the saw-type drum at a linear teeth speed of $7 \mathrm{~m} / \mathrm{s}$, impacts against the flat grate were executed, which was built into the tube sensor with a frequency of its natural vibrations of $3,000 \mathrm{~Hz}$. At the same time, the flat grate was installed at different angles $\gamma_{0}$ with the help of a specially designed rotary device.

The signals were acquired from tensor gauges, attached to the tubular sensor in the form of a half-bridge. These signals were then amplified by an amplifier and sent to a loop oscilloscope with self-developing ultraviolet photographic paper. The signal from the oscillograph was recorded, after the self-development of photographic paper in the light, it was possible to process the study results.

Our research into the use of mounted cleaners on cotton harvesting machines makes it possible to get closer to solving the task of obtaining high-quality machine-harvested raw cotton by reducing the content of litter.

This ensures the high quality of raw cotton both after field cleaning and under the conditions of a cotton gin involving its natural processing based on the shortened technology.

The main drawback of these devices is that it is impossible to determine the cleaning effect of each grate because the weeds released into the gap between the grates are collected in one or more compartments, which is why the experiments do not take into consideration the following:

a) a change in the raw cotton litter content during a transition from one grate to the next one in the process of cleaning;

b) the effect of the gap between the grates, the shape and size of the grates on the cleaning effect of the grate and the grate section.

The variances in these parameters are given in Tables 1,2 . 
The theoretical study of the cleaning process has shown that an important reserve for the process intensification could be a variation in the angle $\gamma$, which indicates that it is possible to revive the fading process of cleaning under the repeated influence of the grates on raw cotton.

\section{Results of studying a grate-saw-like cleaner of large litter of the mounted type on the technological process of cleaning raw cotton}

\section{1. Theoretical study into the effects of grates on weeds in cleaning processes}

Work [9] reported a theoretical model of the cleaning process involving an impact against the grate, which is close to the actual one. A series of assumptions have been adopted: a link between the fly and litter is linear-elastic; a weed particle, lying at the outer open surface of the fly, can execute radial and tangent movements relative to the center - the seed of the fly. At the same time, both movements and the impact force influence are considered to be located in the same plane. That simplifies the problem by reducing the weed particles to a system with one mass $\mathrm{m}_{\mathrm{c}}$ and two orthogonal elastic elements with the stiffness ratios $c_{1}$ and $c_{2}$, exposed at a random angle $\alpha$ to the impact force (Fig. 2).

For this model, in order to move from the known impact force $P(t)$ to the unknown effect on the litter, it is necessary to calculate the transfer function.

Consider the non-steady forced fluctuations of the system in each direction of the $x$ and $y$ axes similar to a system with one degree of freedom:

$$
\begin{aligned}
& x^{\prime \prime}+p_{1} x=\frac{P(t) \sin \alpha}{m c}, \\
& y^{\prime \prime}+p_{2}=\frac{P(t) \cos \alpha}{m c},
\end{aligned}
$$

where $p_{1}$ and $p_{r}$ are, respectively, the frequencies of the natural oscillations of elements in the absence of friction, they are equal to:

$$
\begin{aligned}
& p_{1}=\sqrt{\frac{c_{1}}{m_{c}}}, \\
& p_{2}=\sqrt{\frac{c_{2}}{m_{c}}},
\end{aligned}
$$

considering

$$
P(t)=F_{0} \sin \alpha .
$$

The total value of the maximum movement of a weed particle along a given direction is to be determined if one designates:

$$
\begin{aligned}
& q_{\max }=x_{\max } \cdot V \cdot y_{\max }, \\
& q_{\max }=\frac{F_{0} \pi}{m_{c} t_{y} P\left(P^{2}-\frac{\pi^{2}}{t_{y}}\right)} \sqrt{2(1-\cos \alpha) t_{y}} .
\end{aligned}
$$

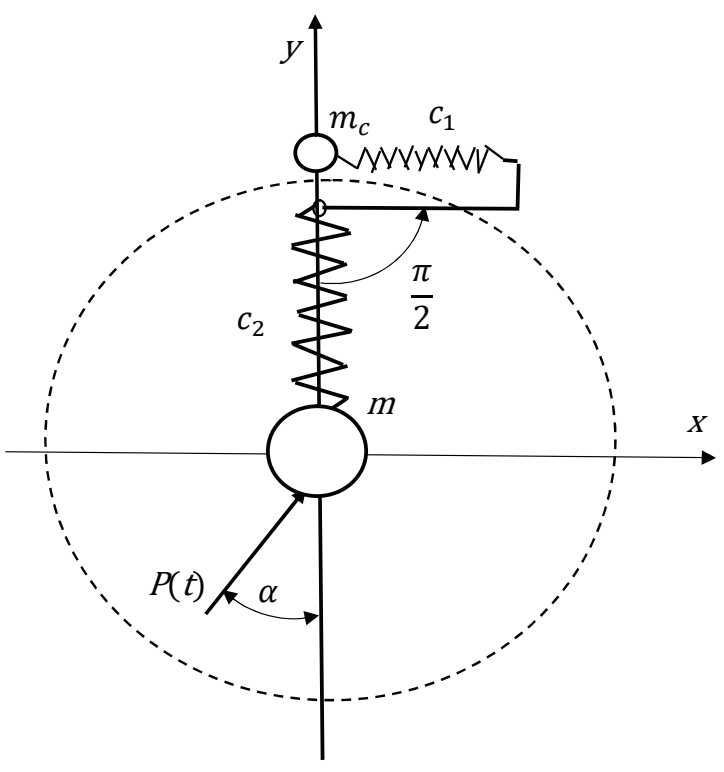

Fig. 2. A model of the transmission of the dynamic effect to a weed particle through raw cotton

At the maximum displacement $q_{\max }$, a weed particle is exposed to the maximal effect exerted by an elastic element along the predefined direction, defined as follows:

$$
R_{\max }=c q_{\max } .
$$

By designating the transfer function via $M$ and by defining it as the ratio of the maximum force quantities $R_{\max }$ and $F_{0}$, taking into consideration formulae (3) to (5), we obtain:

$$
M=\frac{R_{\max }}{F_{0}} .
$$

The existing cleaning process does not make it possible to control the values of $t_{y}, c$, and $m_{c}$, and, therefore, the $M_{c}$ quantity. The necessary cleaning effect is obtained by increasing the impact force $F_{o}$, that is due to loosening the bonds between the litter and fiber by changing the random angle $\alpha$.

And, at the same time, as a result of repeated influences, the bond between the litter and fiber would be somewhat differently (due to a change in the direction of force) loosened. Taking into consideration the possibility of moving a weed particle along the $x$ and $y$ direction at $c=c_{1}$ and $c=c_{2}$, respectively, we could obtain the value for the transfer function $M_{x}$ and $M_{y}$ when fitting rigidity values in formula (6).

Given the non-simultaneous nature of the maximum influence of both elastic elements, their geometric sum is determined as follows:

$$
R_{\max }=P_{\max } \sqrt{M_{x}^{2} \sin ^{2}+M_{y}^{2} \cos ^{2} \alpha} .
$$

At $\alpha=0$ and $\alpha=\pi / 2$, weed particles are exposed to both radial or tangential elements, and the weed particles come off the surface of a cotton particle in radial or tangent directions.

The values of $M_{x}$ and $M_{y}$ are random; each has its own statistics, which depend on the breed grade of cotton, the degree of its maturity, which significantly affects $c$, the raw cotton moisture content. As a result, at the same value of the external influence $P_{\max }$, the angle $\alpha$, and the constant time of the impact, the weed particles of the same mass $m_{c}$ would be exposed to a different magnitude of the force $F_{0}$. 
In addition, cotton has different $\underline{m}_{c}$ and $\alpha$ statistics. For a given value of $\bar{a}_{1}, \bar{c}_{1}, \bar{c}_{2}, \bar{m}, \bar{t}_{y}$ at a mean value of the maximal impact force $P_{\max }$, we can note the mean reaction $R_{\max }$ with respect to the mean value of dynamic strength.

The $r$ value, closely related to the dynamics of the cleaning process and the physical-mechanical properties, should be larger and, at $r>1$, provide for the removal of litter from the open surfaces of structural particles.

The cleaning process is not limited to a single load application as discussed above. Moreover, the re-application of force $P(t)$ can in all respects coincide with the previous one in order to loosen the strength of the bond $F_{s c}^{n}$ with the litter not removed from the cotton earlier. This is done in the section of large litter cleaner with consistent interaction with the identically shaped and placed grates.

If the model considered assumes the constancy of the characteristics of rigidity, mass, it is obvious that in the interaction with any successive grate the impact force can be considered constant.

At the same time, the criterion of cleaning process efficiency increases with an increase in the number of impacts, which means a decrease in the average strength of the fiber grip with litter during repeated influences. Physically, it is a statistical law: unlike a single exposure with a small number of load cycles, a decrease in the average strength of the fiber grip with litter can be achieved. This occurs in the section of large litter cleaner in the consistent interaction with the identically shaped and placed grates.

Given the variability in $F_{s}$, with an increase in the number of influences $n$ from a certain starting value to a limit, tending to zero or some constant quantity, it is possible to adopt this very model of the transmission of dynamic influence to a weed particle through raw cotton (Fig. 2).

The process exponentiality corresponds to the nature of the phenomenon and the static nature of it:

$$
\overline{F_{s c}^{n}}=F_{s c}\left[S-(S-1) \cdot e^{-\beta(n-1)}\right]^{-1},
$$

where $S, \beta$ are the constants of a two-parametric exponent; $n$ is the number of cycles of exposure to the same type of working bodies on raw cotton.

At $n=1, \overline{F_{s c}^{n}}=\overline{F_{s c}}$ at $n \rightarrow \infty \overline{F_{s c}^{n}}=\frac{\overline{F_{s c}}}{S}$, that is, it means a multiplier of grip strength from $n=1$ to $n \rightarrow \infty$ and can reach large values.

Considering (8), the expression from [10] is rewritten for $\bar{r}_{n}$ as follows:

$$
\overline{r_{n}}=\frac{P_{\max }}{\overline{F_{s c}}}\left[S-(S-1) e^{-\beta(n-1)}\right] \sqrt{M_{x}^{2} \sin ^{2} \alpha+M_{y}^{2} \cos ^{2} \alpha} .
$$

_ It is obvious from formula (9) that with a growth in $\bar{n}$ the value of $\overline{r_{n}}$ increases, and, at some value of $n$, can reach the value that provides for the necessary degree of raw cotton cleaning.

The change can reach the variable of the average $\alpha$ value that is achieved in existing cleaners when the cleaned material moves from one $\bar{r}_{n}$ section to another.

In order to implement this idea and revitalize the process to change the monotony of the influence $\alpha$, we propose grates with different locations of the front face (angle $\gamma_{0}$ ) relative to the saw-type drum.
It is important to assess how much the tilt of the grate and its variation affects the condition for the removal of litter from the cleaning area and other dynamic characteristics (the force of impact).

\section{2. Estimating the dynamic characteristics of a saw-} type-grate section

It is known that the impact interaction between material bodies is characterized by the magnitude of pulse $S$, a comprehensive dynamic characteristic that is an integral

$$
S=\int_{0}^{t} y(t) \mathrm{d} t,
$$

where $P(t)$ is the force of impact in a time function $t ; t_{y}$ is the time of impact.

The impact to solids refers to a dynamic process whose duration is less than 0.0001 seconds, and can even last for millionths of a second.

Formula (10) is used in the elementary theory of impact when the time of impact is practically impossible or very difficult to measure. In these cases, one describes the state of the system before and after the impact, using the known recovery factor $K_{y}$ as an internal characteristic of energy dissipation during an impact.

This theory does not make it possible to estimate such important characteristics as the time of impact $t_{y}$ and the maximum impact force $P_{\max }$. The application to cotton of Hertz theory, which takes into consideration internal phenomena, as well as wave theory, is problematic.

According to data given in [1], the time of the fly's impact against the grate or pin is $0.0025-0.006 \mathrm{~s}$, which makes it inappropriate to use (10) to such problems [13].

In the cited works, the blow of the fly with the grate is considered as an element of the vibrational motion of elastic (linearly or nonlinearly) particles possessing mass $m$ and initial speed $V_{n}$.

And, their authors considered a variety of problems involving several elastic elements, orthogonal or mutually dependent.

It is shown that the main value that controls the impact of a "fly" is the elasticity of the fibrous cover of the seed, which makes it possible, for the practical purpose, to confine ourselves to the simplest linear model (Fig. 3).

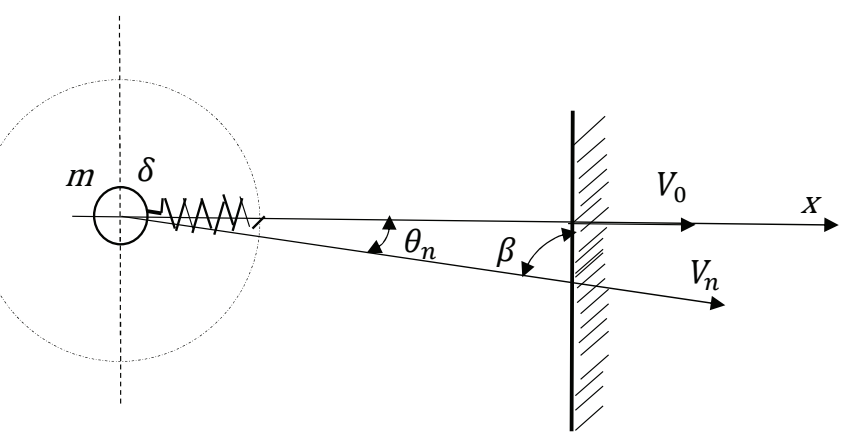

Fig. 3. Linear model of the fly's oblique impact against a still surface

In a general case, the fly is at some angle $\beta$ to the surface. This angle is complemented to the right angle of falling $\theta_{m}$ :

$$
\theta_{n}+\beta=\frac{\pi}{2}
$$


These angles predetermine the speed component that implements the impact $V_{0}$ :

$$
V_{0}=V_{n} \cos \theta_{n}=V_{n} \sin \beta .
$$

Assume the following:

1. A fly is considered linearly elastic with a ratio of malleability $\delta$.

2. Its mass is concentrated in the center.

3 . The time when the elements of the system come into contact is considered much less than the time of the impact. Then, by D'Alembert, the equation of movement along the $x$ axis takes the following form:

$$
m \frac{d^{2} x}{d t^{2}}+\frac{x}{\delta}=0
$$

derived from the condition of equality between the forces of inertia and the reaction of the grate.

By denoting

$$
P=\sqrt{\frac{1}{m \delta}}
$$

we shall transform (14) into a canonical form

$$
\frac{d^{2} x}{d t^{2}}+P^{2} x=0
$$

A solution to (16), as it is known, $P^{2}>0$, takes the following form:

$$
x=A \sin p t+B \cos p t=C \sin (p t+\varphi),
$$

where $A, B, C, \varphi$ are the constants that depend on the initial conditions.

It is obvious from (15) that $p$ is a physical constant termed a circular oscillation frequency related to the $T$ period via the following ratio:

$$
T=\frac{2 \pi}{p} .
$$

Fig. 4 shows that in the accepted model the impact is executed over only half the period of fluctuations, that is

$$
t_{y}=\frac{T}{r}=\frac{\pi}{\rho}=\pi \sqrt{m \delta},
$$

at $t=0$ (before the impact) $x=0$.

From (16), we obtain $B=0, A=\frac{V_{0}}{\rho}$, and the law of displacement takes the following form:

$$
x=\frac{V_{0}}{\rho} \sin p t .
$$

Of interest is not only the law of motion (it is harmonic) but also the maximum of the deformation and force. It is obvious that

$$
x_{\max }=\frac{V_{0}}{\rho}=V_{0} \sqrt{m \delta},
$$

and the impact force is

$$
P_{\max }=\frac{x_{\max }}{\delta}=\frac{V_{0}}{\rho \delta}=V_{0} \sqrt{\frac{m}{\delta}} .
$$

Thus, from (18), (20), and (21), it is obvious that the force of impact, the maximum deformation at impact depend on the mass, the malleability ratio $\delta$, and the initial speed of the impact $V_{0}$.

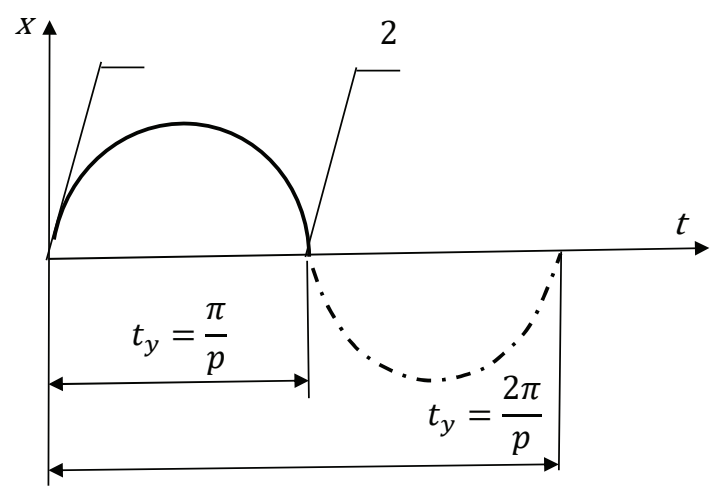

Fig. 4. Characteristic of a fly's elastic deformation during impact: 1 - the fly touches the grate; 2 - the moment the fly detaches from the grate

Therefore, adjusting the dynamic parameters of the impact against a grate is possible only through the variation in $V_{0}$, and not even in the value of $V_{0}$ but the angles at which a fly meets a grate $\beta$ (or $\theta_{n}$ ).

Therefore, we shall rewrite (20), (21) in a more convenient form considering formula (3):

$$
\begin{aligned}
& x_{\max }=V_{n} \sqrt{m \delta} \cos \theta_{n}, \\
& P_{\max }=V_{n} \sqrt{\frac{m}{\delta}} \cos \theta_{n} .
\end{aligned}
$$

The ratio of dynamic rigidity is defined in $[1,3]$ :

$$
\delta=0.00564 \ldots . .0 .0202 \mathrm{~m} / \mathrm{n} \text {. }
$$

To determine $\theta_{n}$, we shall consider the impact of the fly with a radius of $r_{d}$ with the link length $l$, carried by a sawtype drum of radius $R$, against a flat grate (a still surface), forming angle $\gamma_{0}$ with the drum's radius (Fig. 5).

At the same time, the fly moves at speed $V_{n}$ and is deflected from the drum at angle $\varphi_{0}$; it forms angle $\gamma_{0}$ with the drum's radius. As shown in Fig. 5,

$$
\varphi=\varphi_{0}+\frac{\pi}{2}
$$

The radius of the fly center $\rho$ is, according to the cosine theorem:

$$
\rho=\sqrt{l^{2}+R^{2}-2 l R \cos \varphi}=\sqrt{l^{2}+R^{2}+2 l R \sin \varphi_{0}} .
$$

In a simplified form, $\rho$ can be expressed as follows:

$$
\rho=R+l \sin \varphi_{0}
$$


The inclination angle of the grate to the drum's radius at the point of the fly's impact (or the center of the fly) $\gamma$ is equal to (Fig. 6):

$$
\gamma=\gamma_{0}+\eta
$$

where $\eta$ is the angle between the radii passing through the center of the fly and the end of the grate.

Fig. 7 shows that the angle $\eta$ can be determined from the sine theorem:

$$
\frac{l_{1}+\frac{r_{d}}{\operatorname{tg}\left(\pi-\gamma_{0}\right)}}{\sin \eta}=\frac{\rho}{\sin \gamma_{0}},
$$

where $l_{1}$ is the length of the fly's contact path with the grate, whence

$$
\eta=\arcsin \frac{l_{1}+\frac{r_{d}}{\operatorname{tg}\left(\pi-\gamma_{0}\right)}}{\rho}=\sin \gamma_{0} .
$$

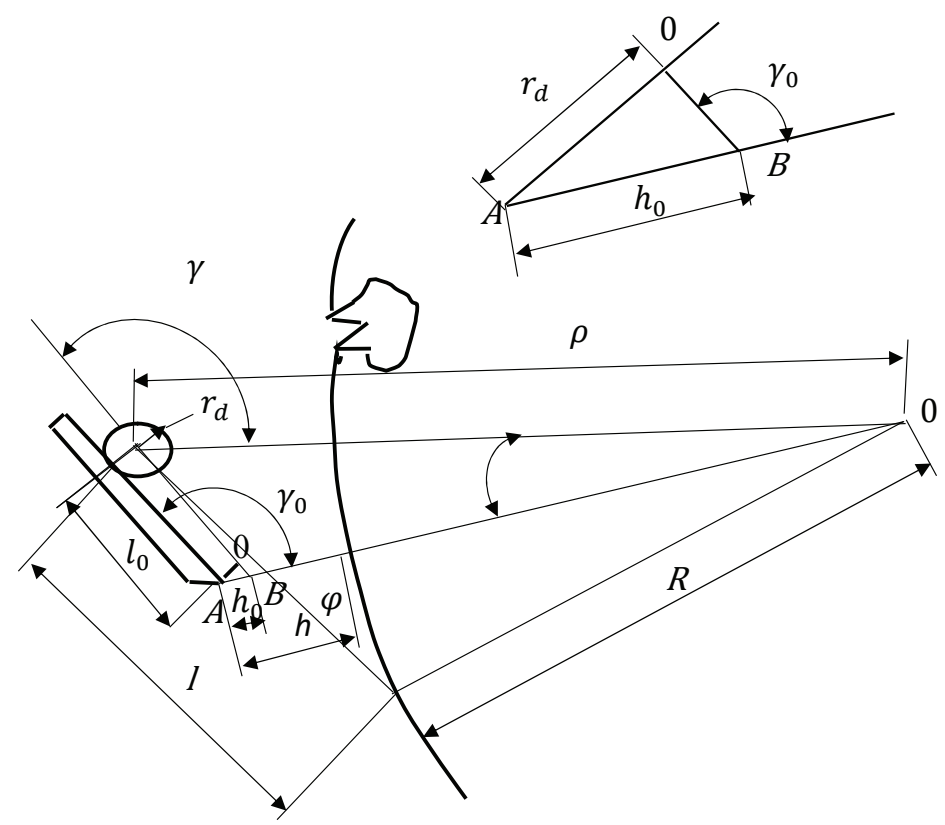

Fig. 5. Scheme of the fly's path over the surface of a flat grate

Considering formula (29),

$$
\gamma=\gamma_{0}+\arcsin \frac{l_{1}+\frac{r_{d}}{\operatorname{tg}\left(\pi-\gamma_{0}\right)}}{\rho} \sin \gamma_{0} .
$$

To determine $\eta$ and $\gamma_{1}$ from formulae (30), (31), one needs to determine $\rho$; to this end, we shall consider $\triangle \mathrm{OBA}$ :

$$
\begin{aligned}
& \rho^{2}=\left(R+h-\frac{r_{d}}{\sin (\pi-\gamma)}\right)^{2}+\left(l_{1}+\frac{r_{d}}{\operatorname{tg}\left(\pi-\gamma_{0}\right)}\right)^{2}- \\
& -2\left(R+h-\frac{r_{d}}{\sin \left(\pi-\gamma_{0}\right)}\right) l_{1}+\frac{r_{d}}{\operatorname{tg}\left(\pi-\gamma_{0}\right)} \cos \gamma_{0} .
\end{aligned}
$$

By solving this square equation without opening brackets and then transforming it, we determine $l_{1}$

$$
\begin{aligned}
& l_{0}=\left(R+h-\frac{r_{d}}{\sin \left(\pi-\gamma_{0}\right)}\right) \cos \gamma_{0} \pm \\
& \pm \sqrt{\left(R+h-\frac{r_{d}}{\operatorname{tg}\left(\pi-\gamma_{0}\right)}\right) \cos ^{2} \gamma_{0}-\left(R+h-\frac{r_{d}}{\sin \left(\pi-\gamma_{0}\right)}\right)^{2}+\rho^{2}-} \\
& -\frac{r_{d}}{\operatorname{tg}\left(\pi-\gamma_{0}\right)}
\end{aligned}
$$

Considering formula (25)

$$
\begin{aligned}
& l_{1}=\left(R+h-\frac{r_{d}}{\sin \left(\pi-\gamma_{0}\right)}\right) \cos \gamma_{0} \pm \\
& \pm \sqrt{\left(R+h-\frac{r_{d}}{\operatorname{tg}\left(\pi-\gamma_{0}\right)}\right)^{2}\left(\cos ^{2} \gamma_{0}-1\right)\left(l^{2}+R^{2}-2 l R \cos \varphi\right)^{2}}- \\
& -\frac{r_{d}}{\operatorname{tg}\left(\pi-\gamma_{0}\right)}
\end{aligned}
$$

We shall determine the value of $l_{1}$ at the following data: $R=240 \mathrm{~mm}, l=\mathrm{mm}, r_{d}=10 \mathrm{~mm}, \gamma_{0}=140 \div 180^{\circ}$, $\varphi_{0}=40 \div 70^{\circ}$ or $\varphi=130 \div 160^{\circ}$ based on data from [14].

The $l_{1}$ values calculated from formula (33) are given in Table 1.

Table 1

The length of the fly's contact arc with the grate depending on a change in $\varphi_{0}$ and $\gamma_{0}$

\begin{tabular}{|c|c|c|c|c|c|}
\hline \multicolumn{6}{|c|}{ The fly's contact path length (mm) } \\
\hline$\varphi_{0}$ & $140^{\circ}$ & $150^{\circ}$ & $160^{\circ}$ & $170^{\circ}$ & $180^{\circ}$ \\
\hline $40_{0}^{\circ}$ & 9.63 & 6.967 & 4.79 & 2.9 & 1.32 \\
\hline $50^{\circ}$ & 12.54 & 9.54 & 7.99 & 5.24 & 3.65 \\
\hline $60^{\circ}$ & 16.56 & 11.67 & 9.25 & 7.56 & 5.51 \\
\hline $70^{\circ}$ & 18.12 & 13.29 & 10.64 & 8.65 & 6.87 \\
\hline
\end{tabular}

As one can see from Table 1, with increasing the inclination angle $\gamma_{0}$ of the grate, $l$ decreases. With an increase in $\varphi_{0}$, that is the gap between the grates at the same values of $\gamma_{0}, l$ increases; as a result, there is an increase in the force of friction. If the saw-type drum carries a fly consisting of more than two pieces, the possibility of overlapping increases, therefore, the removal of the flies increases.

Based on the found values of $l_{1}$, we shall determine the values of angle $\eta$ and $\gamma$ from the respective formulae (29) and (30).

Given that the speed of a fly is perpendicular to the radius of the drum at the moment of impact, taking into consideration Fig. 8, we determine that the angle of the fly's fall is equal to:

$$
\theta_{0}=180^{\circ}-\gamma_{0}
$$

The magnitude of the fly impact force against a grate, calculated from formula (23), is given in Table 2 for the T-1 grade fly with a mass of $m=0.17 \mathrm{~g}$; and for a fly with litter $m_{c}=0.92 \mathrm{~g}$. In this case, $V_{n}=7.8 \mathrm{~m} / \mathrm{s}$.

Table 2 shows that with an increase in the grate's inclination angle, the force of the impact increases; at $\gamma=180^{\circ}$, it accepts the maximum value, that is, a direct impact of the fly 
is executed. The lowest values of force from $\varphi_{0}$ are insignificant, so we provide data for $\varphi_{0}=40^{\circ}$ and $\varphi_{0}=50^{\circ}$.

Table 2

The fly's impact force value

\begin{tabular}{|c|c|c|c|c|}
\hline \multicolumn{3}{|c|}{ Angle value } & \multicolumn{2}{c|}{$P_{\max }$} \\
\hline \multirow{4}{*}{$\varphi_{0}$} & $\gamma_{0}$ & $\Theta_{0}$ & at $m$ & at $m_{c}$ \\
\hline \multirow{4}{*}{$40^{\circ}$} & $140^{\circ}$ & $36^{\circ}$ & $0.56-1.12$ & $0.89-1.78$ \\
\cline { 2 - 5 } & $160^{\circ}$ & $17^{\circ}$ & $0.62-1.23$ & $0.98-1.97$ \\
\cline { 2 - 5 } & $170^{\circ}$ & $7^{\circ}$ & $0.69-1.38$ & $1.10-2.2$ \\
\cline { 2 - 5 } & $180^{\circ}$ & 0 & $0.7-1.39$ & $1.12-2.23$ \\
\hline \multirow{5}{*}{$50^{\circ}$} & $140^{\circ}$ & $36^{\circ}$ & $0.56-1.12$ & $0.89-1.76$ \\
\cline { 2 - 5 } & $160^{\circ}$ & $26^{\circ}$ & $0.62-1.33$ & $1.06-2.12$ \\
\cline { 2 - 5 } & $170^{\circ}$ & $20^{\circ}$ & $0.76-1.38$ & $1.10-2.2$ \\
\cline { 2 - 5 } & $180^{\circ}$ & 0 & $0.7-1.39$ & $1.11-2.22$ \\
\hline
\end{tabular}

\section{3. The influence of the grate's inclination angle on} its cleaning effect

As we know from [8], the litter that hits a still surface, together with a fly at speed $V_{n}$ at angle $\theta_{n}$, is repelled from it, changing its direction and magnitude at speed $V_{\text {om }}$ and at angle $\theta_{\text {om }}$ (Fig. 6).

At the same time, the tangents that make up the speeds of falling and repelling are almost the same, and their value is equal to:

$$
V_{r}=V_{n} \sin \theta_{n}
$$

while the normal one is damped by the value proportional to the recovery rate $K_{y}$, then

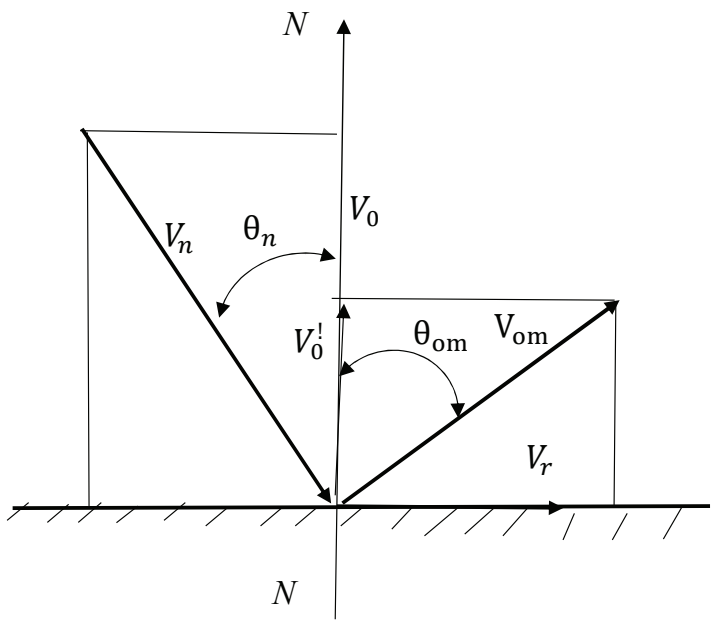

Fig. 6. Estimation of kinematic characteristics before and after an impact

$$
V^{\prime}=V_{0} \cdot \cos \theta_{n} \cdot K_{y} .
$$

Fig. 6 shows that the tangent of the inclination angle is equal to

$$
\operatorname{tg} \theta_{o m}=\frac{V_{r}}{V_{0} \cdot K_{y}}=\frac{\operatorname{tg} \theta_{m}}{K_{y}},
$$

whence

$$
\operatorname{tg} \theta_{o m}=\operatorname{arc} \frac{V_{r}}{V_{0} \cdot K_{y}}=\operatorname{arctg} \frac{\operatorname{tg} \theta_{m}}{K_{y}} .
$$

The absolute speed value is:

$$
V_{\text {om }}=\sqrt{V_{r}^{2}+V_{0} K_{y}^{2}}=V_{n} \sqrt{\sin ^{2} \theta_{n}+K_{y}^{2} \cos ^{2} \theta_{n}} .
$$

Determine the values of $\theta_{o m}$ and $V_{o m}$ for different $\gamma_{0}$ from formulae (38) and (39), taking into consideration that $V_{k}=$ $=7.6 \mathrm{~m} / \mathrm{s}$ at $\varphi_{0}=50^{\circ}$ (Fig. 8). We calculate for $K_{y}=0.1 \div 0.4$, taking into consideration a change in the mass and recovery rate of weeds although, according to data given in $[5,8], K_{y}=0.33$.

Based on the found values, we build a dependence chart $V_{\text {om }}=f\left(\gamma_{0}\right)$ (Fig. 7) and $\theta_{n}=f\left(\gamma_{0}\right)$ (Fig. 8) for various values of $K_{y}$ where plots I-V correspond to $K_{y}=0.1 ; 0.2 ; 0.3 ; 0.33 ; 0.4$.

As Fig. 7 shows, an increase in $\gamma_{0}$ leads to a decrease in $V_{o m}$ at all $K_{y}$

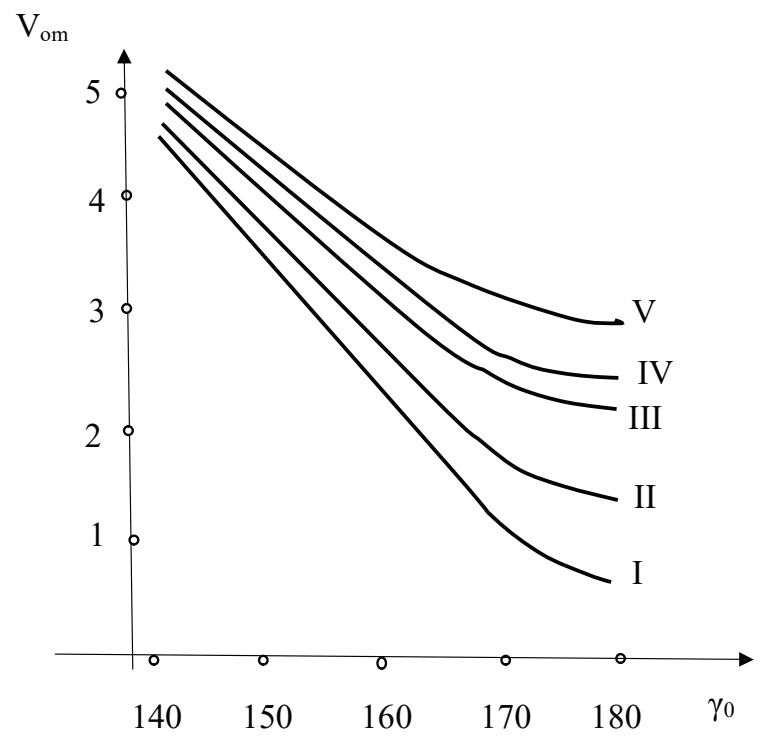

Fig. 7. Dependence chart of rebound speed $V_{o m}$ on angle $\gamma$

At the same time, up to $\gamma_{0}=165^{\circ}$, this change is almost linear.

With an increase in $K_{y}$ at the same angles $\gamma_{0}, V_{o m}$ increases. Fig. 6 shows that an increase in $\gamma_{0}$ leads to a decrease in $\theta_{\text {om }}$ for all $K_{y}$.

The charts in Fig. 7, 8 show that a decrease in $\gamma_{0}, V_{o m}$, and $\theta_{o m}$ leads to an increase in the possibility of the removal of weeds. However, this reduces the force of the fly's impact against a grate, as well as, therefore, the possibility of breaking a bond between the weed and fly, which is undesirable. At $\gamma_{0}=180^{\circ} \theta_{o m}=0$ for all $K_{y}$, there is a slip of the weed particles over the plane of the grate. As one can see from Fig. 8 , the maximum value of the inclination angle $\gamma$, at which a weed particle is repelled, is $\gamma_{0}=175^{\circ}$.

These data are given in Table 3.

As one can see from Table 3 , as the values of the angles $\varphi_{0}$ increase, $\gamma_{0} \theta_{n}$ decreases. Moreover, with a change in $\gamma_{0}$ the value of $\theta_{n}$ changes significantly; at $\gamma_{0}=180^{\circ}, \theta_{n m}=0$, for $\varphi_{0}-$ slightly.

After a weed particle is repelled at speed $V_{\text {om }}$, the gravity $G=m q$ and the air drag $S_{b}$ (Fig. 9) act on it during the flight.

Determine the trajectory of a weed particle's flight without taking into consideration the force of aerodynamic resistance (if it is taken into consideration, the solution to the problem would be much more complicated). 


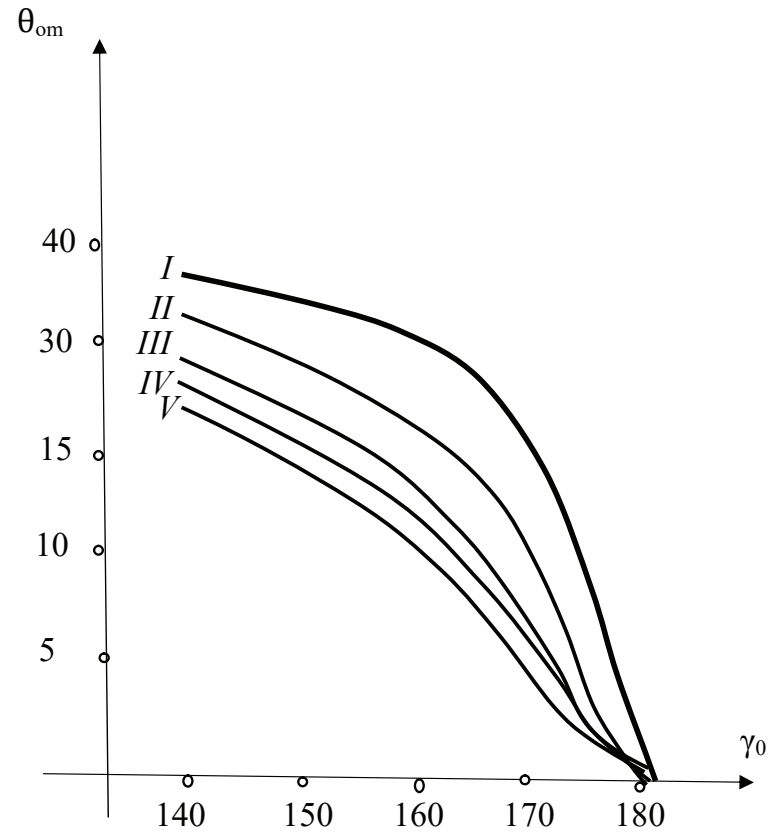

Fig. 8. Dependence chart of rebound angle $\theta_{o m}$ on angle $\gamma_{0}$ at different values of the recovery factor $K_{y}$

Table 3

The value of fall angle $\theta_{n}$ depending on $\gamma_{0}$ and $\varphi_{0}$

\begin{tabular}{|c|c|c|c|c|}
\hline \multicolumn{4}{|c|}{ Angle value } & \multicolumn{4}{|c|}{$\theta_{n}$} \\
\hline$\varphi_{0}$ & $40^{\circ}$ & $50^{\circ}$ & $60^{\circ}$ & $70^{\circ}$ \\
\hline $140^{\circ}$ & $36^{\circ} 87$ & $36^{\circ} 48$ & $35^{\circ} 90$ & $35^{\circ} 67$ \\
\hline $145^{\circ}$ & $32^{\circ} 00$ & $3148^{\circ}$ & $31^{\circ} 50$ & $31^{\circ} 35$ \\
\hline $150^{\circ}$ & $2726^{\circ}$ & $299^{\circ}$ & $2678^{\circ}$ & $26^{\circ} 63$ \\
\hline $155^{\circ}$ & $2240^{\circ}$ & $2218^{\circ}$ & $22^{\circ} 01$ & $21^{\circ} 09$ \\
\hline $160^{\circ}$ & $20^{\circ} 44$ & $20^{\circ} 25$ & $17^{\circ} 21$ & $17^{\circ} 13$ \\
\hline $165^{\circ}$ & $17^{\circ} 51$ & $17^{\circ} 34$ & $12^{\circ} 39$ & $1232^{\circ}$ \\
\hline 170 & $7^{\circ} 62$ & $7^{\circ} 48$ & $7^{\circ} 54$ & $7^{\circ} 5$ \\
\hline 175 & $2^{\circ} 66$ & $2^{\circ} 59$ & $2^{\circ} 07$ & $2^{\circ} 65$ \\
\hline 180 & 0 & 0 & 0 & 0 \\
\hline
\end{tabular}

Taking the rebound point for the coordinate origin, consider the movements of a weed particle relative to the horizon (Fig. 9). The position of the weed particle at any time $t$ is determined by the following coordinates:

$$
\begin{aligned}
& x=V_{\text {om }} t \cdot \cos \theta_{0}, \\
& y=V_{\text {om }} t \cdot \sin \theta_{0}-\frac{q t^{2}}{2},
\end{aligned}
$$

and the trajectory equation is written in the following form

$$
y=x \operatorname{tg} \theta_{0}-x^{2} \frac{q}{2 V_{o m}^{2} \cos ^{2} \theta_{0}}
$$

where $\theta_{0}$ is the angle of the weed particle discharge.

As shown in equation (41), the trajectory of the weed particle is a parabola.

At the same $V_{\text {om }}$, the shape of the trajectory depends on the angle $\theta_{0}$.

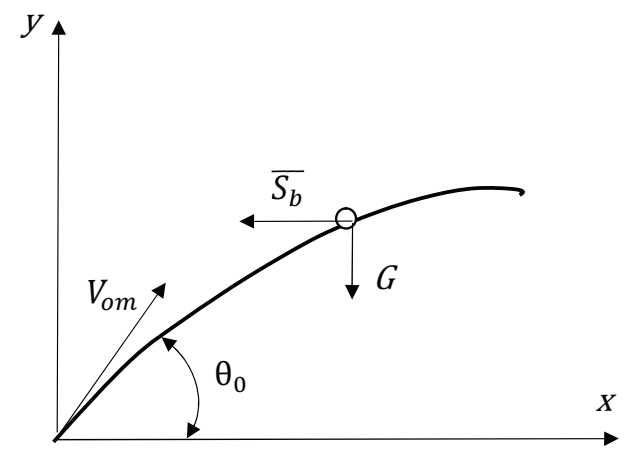

Fig. 9. Scheme of the trajectory of the free flight of a weed particle after an impact

According to data in (5), and, as seen in Fig. 8, this angle is equal to:

$$
\theta_{0}=\gamma-90^{\circ}-\theta_{o m}-\alpha
$$

where $\alpha$ is the central angle that determines the position of the grate relative to the horizontal axis running through the center of the drum.

Taking into consideration the value of the angle $\gamma_{0}$ :

$$
\theta_{0}=\gamma_{0}+\eta-90^{\circ}-\theta_{\text {om }}-\alpha
$$

The $\theta_{o m}$ curves, depending on the data for $\gamma_{0}, \varphi_{0}$, are shown in Fig. 8 for different values of $K_{y}$.

As the angle $\alpha_{0}$ increases, the $\theta_{0}$ value decreases and takes a negative value. The smaller $K_{y}$, the earlier it happens.

For the grates located at the bottom of the horizontal axis and moving away from it, $\theta_{0}$ increases.

As one can see from Fig. 10, the weed particles are thrown in the direction of the saw-type drum. At the same time, the weed particles are partially carried away by airflow into the inter-grate gap, as well as discharged by centrifugal force into one of the following gaps between the grates. This phenomenon occurs if a weed particle is thrown up from the horizon, and the direction of the flight would be in the opposite direction to the drum movement.

If the direction of the flight is downwards from the horizontal, that is, towards the movement of the drum, the weed particles are pressed to the saw-type surface by a fly when passing the gap between the grate and the drum, that is, they penetrate the fibrous mass again.

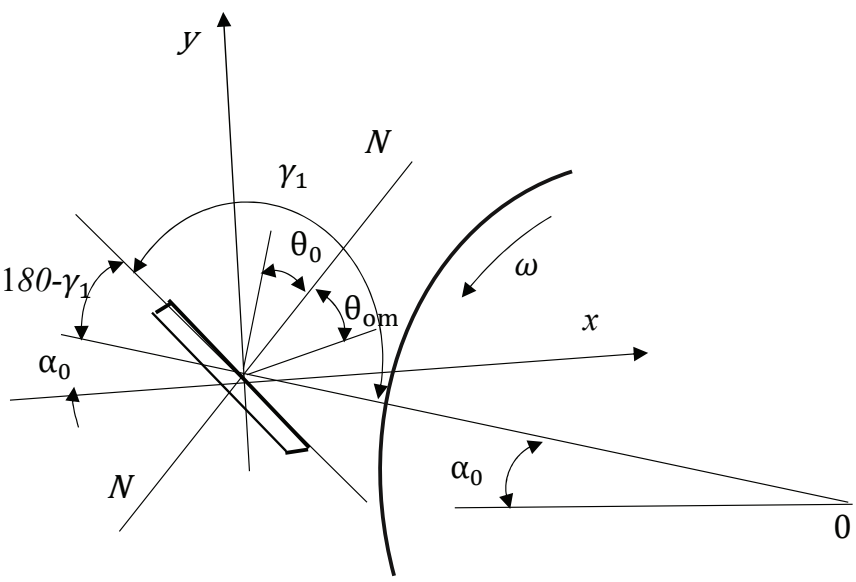

Fig. 10. Scheme to determine the angle of a weed particle's removal 
In addition, the fly closes the possible way for litter when it is removed by centrifugal force in subsequent gaps.

It follows that for the grates located above the horizontal axis, their inclination angle $\gamma_{0}$ should increase in proportion to the distance, that is when $\alpha_{0}$ increases. This fact was taken into consideration in the design of the grate for a mounted cleaner.

\section{Discussion of results of studying the effect of a mounted cleaner on the cleaning process}

The applied theoretical methods for studying the effect of grates do not contradict the elementary theory of impact, which makes it possible to qualitatively reveal the essence of the process of energy dissipation when the fly hits the grate. While this phenomenon is characterized by the recovery factor $K_{y}$ in elementary theory, within the elastic model examined in this work this phenomenon is quantitatively associated with viscous resistance $\left(K_{v z}\right)$, the time of impact $t_{\mathrm{y}}$, as well as the values of $\gamma_{o}$ and $\delta$.

Our analysis is crucial for assessing the technological characteristics of the grates in a cleaning section of large-litter cleaners. This makes it possible to give a theoretical assessment of the repelling ability of the grates in large-litter cleaners with flat faces, to assess the space across which the repelled weed particles and cotton flies move after having lost contact with the saw-type drum.

Given the development of spindle harvesting of raw cotton and the deterioration in its quality, intensive work is underway to clean cotton from large and small weeds. However, the successful solution to the problem is hampered by the high speed of transporting cotton in the pneumatic cleaner, which leads to its damage; this problem is complicated by a series of structural issues related to the difficulty of arranging cleaning units on a cotton harvester, the need to effectively regenerate raw cotton and reduce its losses. These problems cannot be successfully solved because until recently there were no theoretical foundations for the design of mounted field cleaners.

The disadvantage of existing cleaners was the increased removal of cotton flies and a low cleaning effect. However, as a result of the introduction of mounted field cleaners, whose cleaning effect reached up to $50 \%$ in the technological process in a cotton gin, the quality of the fiber has improved with the number of defects decreased by $10-20 \%$. When using mounted cleaners, the following technological cleaning scheme was recommended: two cleaners KhChE, installed in parallel, and 5-6 BCh-2M cleaners, the screw cleaner 6A-12M manufactured in the Uzbek Republic.

This could provide for the measures that need to be taken to remove litter from the area of the grate.

We have experimentally investigated the impact interaction between the cleaner's saw teeth and raw cotton particles. The time of build-up and the magnitude of the impact load have been determined, which are $0.0025-0.006 \mathrm{~s}$ and
$0.60-1.30 \mathrm{n}$, respectively, at a change in the speed of raw cotton fly from 7.8 to $12.8 \mathrm{~m} / \mathrm{s}$ (Table 2 ).

The equations (18), (20), and (21) that we built clearly indicate that the force of the impact, the maximum deformation at impact depend on the mass, the malleability ratio $\delta$, and the initial speed of the impact $V_{0}$.

Table 1 demonstrates that as the inclination angle of the grate $\gamma_{0}$ increases, the value of $l$ decreases. With an increase in $\varphi_{0}$, that is, in the gap between the grates at the same values of $\gamma_{0}, l$ increases; as a result, there is an increase in the force of friction.

The nature of the phenomenon has been revealed on the basis of a linear elastic-plastic model when the angle of fall is less than the angle of repelling and is associated with energy losses at impact, that is, depends on the recovery rate $K_{y}$.

Based on the theoretical studies of (37) to (39), a diagram of the trajectories of the litter repelled by the grates (Fig. 6) was considered. It is shown that at no angle $\gamma$ it is possible to directly remove the litter without returning it to the surface of the saw-type drum.

Table 3 gives the values of the fall angle $\theta_{n}$ depending on $\gamma_{0}$ and $\varphi_{0}$, which show that as the values of angles $\varphi_{0}$ and $\gamma_{0}$ increase, the $\theta_{n}$ angle decreases. Moreover, when $\gamma_{0}$ changes, the value of $\theta_{n}$ changes significantly, and, at $\gamma_{0}=180^{\circ}, \theta_{n}=0$; at $\varphi_{0}-$ slightly.

We have considered the mechanics of the process of interaction between a raw cotton fly and the working bodies in the section of a large-litter cleaner of the mounted type. The processes of fixing and removing raw cotton from the saw-type gear have been investigated, as well as pulses of the impact interaction between the flies and the grate. The dynamics of the process have been brought to the calculation of values for the impact pulse. At the same time, it is necessary to move to the magnitude of the impact force, requiring the knowledge of the characteristics of the dynamic rigidity of the material and the construction of a model that would be adequate in its essence to the scheme under consideration.

\section{Conclusions}

1. The theoretical model of the generalized cleaning process has shown that an important reserve for process intensification could be a variation in the angle of the grate, which would make it possible to revive the fading cleaning process at a repeated influence of the grates on raw cotton.

2 . Based on the linear elastic-plastic model, the nature of the impact process of the interaction between a fly and a grate has been revealed, when the angle of fall is less than the angle of repelling and is a function of energy losses at impact, that is, depending on the recovery factor.

3. It has been established that no inclination angle of the grate could make it possible to directly remove the litter from a technological flow without its temporary return to the surface of the saw-type drum.

\section{References}

1. Tyutin, P. N., Lugachev, L. E. (1997). O vydelenii sornyh primesey cherez yacheyki setchatyh poverhnostey. Sbornik trudov TITLP "Mekhanicheskaya tekhnologiya voloknistyh materialov ", 19, 51-58.

2. Matusiak, M., Walawska, A. (2010). Important Aspects of Cotton Colour Measurement. FIBRES \& TEXTILES in Eastern Europe, $18(3(80)), 17-23$. 
3. Boldinskiy, G. I., Samandarov, S. A., Benenson, A. L. (1974). Vybor formy kolka v ochistitelyah melkogo sora. Hlopkovaya promyshlennost', 1, 16-17.

4. Baker, R. V., Stedronsky, V. L. (1998). Seed cotton and cottonseed handling wish air yet conveyrs. U. S. Department of agriculture, 18.

5. Korabel'nikov, R. V., Ibrogimov, H. I. (2008). Kompleksnyy pokazatel' vozdeystviya ochistitelya hlopka na hlopok-syrets v protsesse ochistki. Tekhnologiya tekstil'noy promyshlennosti, 3, 35-38.

6. Mirochnichenko, G. I., Korabelnikov, R. V., Jakubov, D., Tjutin, P. N. Pat. No. 1.475.448. Great Britain and Northern Ireland.

7. Shaw, G. S, Franks, S. N. (1994). Hand book for cotton Gunners. Washington, 28-32.

8. Hafizov, I. K., Rasulov, A. (2009). Issledovanie razryhlitel'nogo effekta razdelitelya dolek tonkovoloknistogo hlopka-syrtsa na letuchki. Hlopkovaya promyshlennost', 3, 9-21.

9. Sapon, A. L., Samandarov, S. A., Libster, S. L. (2007). Potochnaya liniya pervichnoy pererabotki hlopka-syrtsa PLPH. Hlopkovaya promyshlennost', $3,1-3$.

10. Ibrogimov, H. I., Korabel'nikov, R. V. (2009). Osobennosti vzaimodeystviya kolkov rabochego barabana ochistitelya s chastitsami hlopka-syrtsa, imeyushchimi voloknistye svyazi, pri netsentral'nom udare. Tekhnologiya tekstil'noy promyshlennosti, 2, 16-19. Available at: https://tp.ivgpu.com/wp-content/uploads/2015/11/314_6.pdf

11. Lebedev, D. A., Petrov, A. A. (2013). Model impacts on weed admixture in the process of cleaning fiber. Tekhnologiya tekstil'noy promyshlennosti, 3, 115-119.

12. Khakimov, Sh. Sh. (2015). Theoretical studies of the motion of raw cotton the gaps between the grate fixing and serated drum. European applied sciences, 11, 63-66.

13. Lin, H., Akankwasa, N. T., Wang, J., Zhang, C. (2019). Simulation of the Effect of Geometric Parameters of the Fibre Transport Channel in Open-End Rotor Spinning. Fibres and Textiles in Eastern Europe, 27 (2 (134)), 52-57. doi: https://doi.org/ 10.5604/01.3001.0012.9987

14. Wang, L., Parnell, C. B., Shaw, B. W. (2002). Performance Characteristics of Cyclones in Cotton-Gin Dust Removal. Agricultural Engineering International: the CIGR Journal of Scientific Research and Development, IV. Available at: https://ecommons.cornell.edu/ bitstream/handle/1813/10269/BC\%2002\%20001.pdf?sequence=1\&isAllowed=y 